\title{
Dominance of the Unaffected Hemisphere Motor Network and Its Role in the Behavior of Chronic Stroke Survivors
}

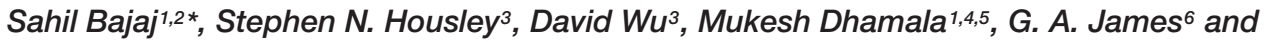 \\ Andrew J. Butler $3,5,7$
}

\begin{abstract}
'Department of Physics and Astronomy, Georgia State University, Atlanta, GA, USA, ${ }^{2}$ Department of Psychiatry, College of Medicine, University of Arizona, Tucson, AZ, USA, ${ }^{3}$ Byrdine F. Lewis School of Nursing and Health Professions, Georgia State University, Atlanta, GA, USA, ${ }^{4}$ Joint Center for Advanced Brain Imaging, Center for Behavioral Neuroscience, Center for Nano-Optics, Center for Diagnostics and Therapeutics, Georgia State University, Atlanta, GA, USA, ${ }^{5}$ Neuroscience Institute, Georgia State University, Atlanta, GA, USA, ${ }^{6}$ Psychiatric Research Institute, University of Arkansas for Medical Sciences, Little Rock, AR, USA, ${ }^{7}$ Department of Veterans Affairs, Atlanta Rehabilitation Research and Development Center of Excellence, Decatur, GA, USA
\end{abstract}

\section{OPEN ACCESS}

Edited by:

Jean-Claude Baron, University of Cambridge, UK

Reviewed by: Nikhil Sharma, University College London (UCL), UK Filippo Brighina, University of Palermo, Italy

*Correspondence: Sahil Bajaj sahil.neuro@gmail.com

Received: 07 September 2016 Accepted: 08 December 2016 Published: 27 December 2016

Citation:

Bajaj S, Housley SN, Wu D,

Dhamala M, James GA and Butler AJ (2016) Dominance of the

Unaffected Hemisphere Motor Network and Its Role in the Behavior

of Chronic Stroke Survivors.

Front. Hum. Neurosci. 10:650. doi: 10.3389/fnhum.2016.00650
Balance of motor network activity between the two brain hemispheres after stroke is crucial for functional recovery. Several studies have extensively studied the role of the affected brain hemisphere to better understand changes in motor network activity following stroke. Very few studies have examined the role of the unaffected brain hemisphere and confirmed the test-retest reliability of connectivity measures on unaffected hemisphere. We recorded blood oxygenation level dependent functional magnetic resonance imaging (fMRI) signals from nine stroke survivors with hemiparesis of the left or right hand. Participants performed a motor execution task with affected hand, unaffected hand, and both hands simultaneously. Participants returned for a repeat $\mathrm{fMRl}$ scan 1 week later. Using dynamic causal modeling (DCM), we evaluated effective connectivity among three motor areas: the primary motor area (M1), the premotor cortex (PMC) and the supplementary motor area for the affected and unaffected hemispheres separately. Five participants' manual motor ability was assessed by Fugl-Meyer Motor Assessment scores and root-mean square error of participants' tracking ability during a robot-assisted game. We found (i) that the task performance with the affected hand resulted in strengthening of the connectivity pattern for unaffected hemisphere, (ii) an identical network of the unaffected hemisphere when participants performed the task with their unaffected hand, and (iii) the pattern of directional connectivity observed in the affected hemisphere was identical for tasks using the affected hand only or both hands. Furthermore, paired $t$-test comparison found no significant differences in connectivity strength for any path when compared with one-week follow-up. Brain-behavior linear correlation analysis showed that the connectivity patterns in the unaffected hemisphere more accurately reflected the behavioral conditions than the connectivity patterns in the affected hemisphere. Above findings enrich our knowledge of unaffected brain hemisphere following stroke, which further strengthens our neurobiological understanding of stroke-affected brain and can help to effectively identify and apply stroke-treatments.

Keywords: functional magnetic resonance imaging (fMRI), effective connectivity, dynamic causal modeling (DCM), motor task, stroke, affected hemisphere, unaffected hemisphere 


\section{INTRODUCTION}

An estimated 795,000 Americans suffer a stroke annually, leading to long-term disability for an estimated 6.4 million Americans. Many stroke survivors exhibit some degree of motor impairment that limits functional status after stroke. Advances in acute care medicine have significantly reduced mortality, which has coincidentally led to rising numbers of stroke survivors that utilize rehabilitation therapies. As the body of evidence of stroke rehabilitation is expanding (Dobkin, 2004; Brewer et al., 2013; Bajaj et al., 2015b), it has become exceedingly important to explore how brain networks are influenced following stroke and the role those networks play in functional recovery. A rich neurobiological understanding of the basic principles of strokerecovery will aid in the development of more effective stroke treatments.

Over the past several years, numerous studies have been proposed to better understand the connectivity patterns in motor network of people suffering from stroke. Most of the studies have focused on the basic motor networks directly involved after stroke (before and after stroke treatment) and compared the results with healthy controls. The primary motor area (M1), which is an integral part of basic motor network, due to its association with upper-limb recovery, is the most common target for stroke therapies. Other motor areas such as premotor cortex (PMC) and supplementary motor area (SMA) are functionally and anatomically in close association with M1 and play a crucial role to execute motor tasks (Bajaj et al., 2014, 2015a,b). Previous studies have discussed the role of the motor network in the unaffected hemisphere of stroke patients and its test-retest reliability with time. Although investigating changes in motor network connectivity strength provide important insight into brain reorganization following stroke, few studies assessing these changes are grounded by the functional ability and motor performance outcomes that are important for stroke survivors with residual upper limb impairment (Fong et al., 2001; Arya et al., 2011; Bajaj et al., 2015a). Recently, in a stroke study, Li et al. (2016) observed significant correlations between the connectivity strength and functional ability, implying that the connectivity of ipsilateral M1 may be useful in evaluating and predicting functional ability and motor performance. This is in agreement with other studies (Grefkes and Fink, 2011; Lindenberg et al., 2012; Chen and Schlaug, 2013) that have found changes in cortical network connectivity of stroke patients are associated with impaired functional ability and motor performance. This is an evolving area of research, with most studies associating clinical outcome to a single region of interest (ROI) association, and fewer studies relating outcome to more complex network models (Park et al., 2011). To our knowledge, no studies have previously compared the role that affected and unaffected hemispheres networks play in encoding stroke patients' functional ability while

Abbreviations: AHem-aHand, Affected hemisphere, affected hand; AHem-bHand, Affected hemisphere, both hands (affected and unaffected); UHem-aHand, Unaffected hemisphere, affected hand; UHem-uHand, Unaffected hemisphere, unaffected hand. simultaneously assessing time-dependent test-retest reliability of these outcomes.

The role of unaffected hemisphere in motor recovery has been considered somewhat controversial (Buetefisch, 2015). It has been reported that the neural substrates in the unaffected hemisphere can mediate recovery only when such substrates in the affected hemisphere are significantly damaged (John et al., 2015). In other studies, abnormalities have been reported in the unaffected arm after stroke, which further depends on whether the infarct was in the dominant or non-dominant hemisphere (Colebatch and Gandevia, 1989; Haaland and Harrington, 1989; Jones et al., 1989; Winstein and Pohl, 1995; Haaland et al., 2004). It is hypothesized that the behavioral recovery observed after stroke is supported by the sensorimotor network in the affected hemisphere (Pineiro et al., 2002; Loubinoux et al., 2003; Calautti et al., 2007; Loubinoux, 2007), whereas it is also hypothesized that the unaffected hemisphere may support motor-recovery (O'Shea et al., 2007; Riecker et al., 2010; Rehme et al., 2011). Although a significant ipsilateral activation has been considered as a marker for poor motor recovery (Ward et al., 2003) alternatively, this has been found in motor areas of subacute and chronic stroke patients (Weiller et al., 1992; Seitz et al., 1998; Bütefisch et al., 2005; Lotze et al., 2006; Schaechter and Perdue, 2008).

Reliability of functional and effective connectivity among motor areas and reliability of various neuroimaging tools over time has been another important aspect to consider when assessing cortical mechanism of recovery. The reliability of functional MRI (fMRI) during visual motor tasks in stroke patients has been tested within and between sessions. By comparing interclass correlation coefficients (ICC), withinsession reliability has been reported to be higher than between session reliability, but the overall results reflect that brain activations are reproducible and such research designs could be used for stroke patients (Kimberley et al., 2008b). Using ROI seed-based and ROI correlation matrix approaches, a 1-year testretest reliability of intrinsic connectivity network was confirmed for older adults using fMRI (Guo et al., 2012). This study was found to be consistent with other short-term reliability studies on young (Schwarz and McGonigle, 2011) as well as older controls (Telesford et al., 2010).

In order to better understand the brain connectivity pattern of the affected and unaffected hemispheres while performing the motor execution task, nine stroke survivors underwent fMRI scanning over two sessions with one-week separation. Our goals in this study were to: (a) Explore the brain connectivity pattern for: (i) affected hemisphere during tapping with affected hand only (AHem-aHand) (ii) affected hemisphere during tapping with both hands (affected and unaffected) simultaneously (AHem-bHand) (iii) unaffected hemisphere during tapping with affected hand only (UHem$a H a n d$ ), and (iv) unaffected hemisphere during tapping with unaffected hand only (UHem-uHand); (b) check if bilateral tapping (i.e., tapping with both hands) strengthened the connectivity patterns more in affected hemisphere compared to unilateral tapping (i.e., tapping with affected hand only) (AHem-bHand vs. AHem-aHand); (c) check if unilateral 
tapping with unaffected hand better estimated the connectivity pattern on unaffected hemisphere (UHem-uHand) than the connectivity pattern on affected (AHem-aHand) and unaffected (UHem-aHand) hemispheres while tapping with affected hand; (d) check if brain connectivity parameters were reliable between two sessions of one week apart; and (e) explore the brain-behavior correlations for affected and unaffected hemispheres.

We hypothesized that the:

(1) connectivity pattern would be (a) stronger for AHem-bHand than AHem-aHand (b) stronger for UHem-uHand than for either AHem-aHand or AHem-bHand and (c) weaker and different for UHem-aHand than AHem-aHand.

(2) connectivity strength parameters would significantly (a) positively correlate with FMA scores and (b) negatively correlate with RMSE scores for UHem-uHand only.

Here higher FMA scores and lower RMSE scores represent better performance and vice-versa.

\section{MATERIALS AND METHODS}

\section{Participants}

Stroke survivors between the ages of 45 and 90 with a moderate to severe unilateral ischemic stroke were recruited. Inclusion criteria included persistent hemiparesis as indicated by a score of 1-3 on the motor arm item of the NIH Stroke Scale (Brott et al., 1989) and significant impairment that limited their activities of daily living (ADL). Those with clinically significant comprised mental status within three days of enrollment were excluded. A total of nine participants were enrolled, all of them had heterogeneous stroke locations distributed to either the left $(n=5)$ or right hemispheres $(n=4)$, resulting in hemiparesis of the contralateral side. Participants underwent fMRI of a motor task (described below) during two sessions with a one-week gap between sessions. Data from one participant was excluded from the analysis because the time between sessions was greater than one week. Written consent was obtained from each participant prior to the experiment and all the participants were compensated for their participation and time. The experimental protocol was approved by the Emory Institutional Review Board (IRB).

\section{Tasks}

All the participants were instructed to lie down in the scanner with arms outstretched close to their body. A blockdesign paradigm (total duration $550 \mathrm{~s}$ ) was used during the experiment where participants were instructed to tap with their left hand index finger (LH), right hand index finger $(\mathrm{RH})$, and both hand index fingers $(\mathrm{BH})$ for $20 \mathrm{~s}$. The protocol for this study was set in a way that all stroke patients had both their affected and unaffected hemispheres involved during scanning. For baseline, a passive resting state (Rest) condition for $10 \mathrm{~s}$ was included (i) before the tapping task, (ii) in between two tapping tasks, and (iii) at the end of the task. The same paradigm was repeated six times as following:

(i) Rest-LH-Rest-RH-Rest-BH (1-90 seconds)

(ii) Rest-RH-Rest-LH-Rest-BH (91-180 s)

(iii) Rest-LH-Rest-BH-Rest-RH (181-270 s)

(iv) Rest-RH-Rest-BH-Rest-LH (271-360 s)

(v) Rest-BH-Rest-LH-Rest-RH (361-450 s)

(vi) Rest-BH-Rest-RH-Rest-LH (451-540 s)

(vii) Rest (541-550 seconds)

\section{Behavioral Data}

In addition to imaging data, clinical data was also collected from five of the nine stroke survivors (clinical data was unavailable on the remaining four). Blinded evaluators assessed clinical outcome measures. Functional ability of affected hand was assessed by the upper extremity portion of the Fugl-Meyer Motor Assessment (FMA) Scale. This is a 33-item test with each item scored on a 3 -point ordinal scale that measures motor function and recovery after stroke (Fugl-Meyer et al., 1975). Scores ranged from 0 (no function) to 66 (normal function) (Sanford et al., 1993). The FMA is a reliable and valid tool for measuring UE impairment following stroke (Gladstone et al., 2002).

To evaluate hand and wrist motor performance of affected hand, root mean squared error (RMSE) was used to determine how closely the participants followed a target presented on a computer monitor, where lower RMSE indicates more accurate tracking and improved motor performance (Wulf and Schmidt, 1997; Gentili et al., 2010). Observed and target tracking performances were recorded ( $50 \mathrm{~Hz}$ sampling rate). Waveform tracking error, as assessed by RMSE, was calculated for the first sine waveform by comparing the observed tracking to the target tracking (equation 1), where $y_{i}$ is the observed tracking and $\hat{y}_{i}$ is the target tracking at time/place $i$ for $n$ observations. This was accomplished by sampling approximately the same length block (first $5 \mathrm{~min}$ ) for every participant. The sampling method was chosen to reduce the impact of fatigue on performance following repeated training bouts (Lorist et al., 2002) and attenuate the occurrence of experience-dependent plasticity within the cortical-cerebellar and cortical-striatal neural systems during the fast learning phase (Doyon and Benali, 2005). The first 40-s of each block were discarded to account for system delay and allow the participant to reach a steady-state tracking performance. The remaining time in the block was used for analysis. The overall RMSE for a given session's block was calculated using the following formula:

$$
R M S E_{\text {Overall }}=\sqrt{\frac{\sum_{i=1}^{n}\left(\hat{y}_{i}-y_{i}\right)^{2}}{n}}
$$

Participant demographics, time post stroke, stroke locations, and baseline behavioral data (FMA and RMSE scores) of the stroke patients are summarized in Supplementary Table S1.

\section{Imaging}

MR imaging was performed at Wesley Woods Center of Emory University, Atlanta, GA, USA. Images were 
acquired with a Siemens 3.0 $\mathrm{T}$ Magnetom Trio scanner (Siemens Medical Solutions, Malvern, PA, USA) using a standard quadrature head coil and multi-band sequence $\left(\mathrm{TR} / \mathrm{TE} / \mathrm{FA}=1000 \mathrm{~ms} / 30 \mathrm{~ms} / 65^{\circ}, 550\right.$ measurements for total duration $9 \min 10 \mathrm{~s}$, resolution $=3 \mathrm{~mm} \times 3 \mathrm{~mm} \times 3$ $\mathrm{mm}$ and 52 transversal slices). An anatomical image of each participant was acquired using a 3D magnetizationprepared rapid acquisition gradient echo (MPRAGE) sequence which consisted of 176 sagittal slices of $1 \mathrm{~mm}$-thickness (resolution $=1 \mathrm{~mm} \times 1 \mathrm{~mm}$, in-plane matrix $=256 \times 256$ ) with TR/TE/FA $=2300 \mathrm{~ms} / 2.89 \mathrm{~ms} / 8^{\circ}$. Participants repeated the fMRI task six times (scans) per imaging session and underwent two scanning sessions separated by 1 week.

\section{Data Analysis \\ FMRI Preprocessing}

Functional MRI data were preprocessed by using SPM12 ${ }^{1}$ (Wellcome Trust Centre for Neuroimaging, London). The preprocessing steps involved slice time correction, realignment, normalization, and smoothing. Motion correction to the middle functional scan was performed within participant using a sixparameter rigid-body transformation. Three translational and three rotational motion-parameters were stored and used as nuisance covariates. The mean of the motion-corrected images was then co-registered to the individual structural image using a 12-parameter affine transformation. The images were then spatially normalized to the Montreal Neurological Institute (MNI) template (Mazziotta et al., 1995) by applying a 12parameter affine transformation, followed by a non-linear warping using basis functions (Ashburner and Friston, 1999). Images were subsequently smoothed with an $8-\mathrm{mm}$ isotropic Gaussian kernel and the low-frequency drifts in signal were removed using a standard band-pass-filter with a $128 \mathrm{~s}$ cutoff.

\section{Volumes of Interest (VOIs)}

In this study, we considered basic motor areas (M1, PMC) and the SMA for each participant, which are well known to play a significant role during motor tasks. Hence, we defined six volumes of interest (VOIs): bilateral M1, bilateral PMC, and bilateral SMA in SPM12 using the first Eigen-variate of activations within a sphere of $6 \mathrm{~mm}$ radius. Before applying first Eigen-variate, VOIs were centered at $(-34,-18,52),(36$, $-18,52),(-34,0,56),(34,0,56),(-6,-6,58)$, and $(6,0$, 62 ) in MNI coordinate system for left M1, right M1, left PMC, right PMC, left SMA, and right SMA, respectively (CárdenasMorales et al., 2014; Bajaj et al., 2015a). Before defining VOIs, we performed the standard uni-variate analysis but we did not find consistent/significant brain activations throughout the sample as the participants suffered from stroke and some of the participants had serious difficulty in performing the task, causing insignificant brain activations. In addition to that, since our analysis also involved connectivity in the ipsilateral side of the brain, so significant brain activation was not found consistently.

Hence, all the VOIs were defined by extracting mean timeseries from the same set of voxels across the participants

${ }^{1}$ http://www.fil.ion.ucl.ac.uk/spm/software/spm12/ for each VOI. For that, we avoided any statistical threshold on activity within areas of interest so that extracted and adjusted time-series data remain spatially identical across all the participants (Parker Jones et al., 2013). The participant specific maxima were constrained to lie within twice the width of Gaussian smoothing kernel (Li et al., 2010; Bajaj et al., 2013).

\section{Dynamic Causal Modeling (DCM)}

In this study, we used random effects Bayesian model selection (RFX BMS) and Bayesian model averaging (BMA) approaches implemented in DCM12 in SPM12a package ${ }^{1}$ (Wellcome Trust Centre for Neuroimaging, London). Here by defining a model space constituting eight models, we computed expected and exceedance probability of each model, along with individual connection strength parameters. DCM is based on dynamical systems theory. Using a set of differential equations, DCM aims to describe how observed brain responses are generated. It estimates the directed connectivity among functionally distinct brain areas by using bilinear approximations to the coupled brain states and further models the influence of external inputs directly to the brain areas or to the connections between functionally distinct areas (Friston et al., 2003).

The task is done by constructing a model space, constituting a set of models where each model represents a set of intrinsic connections among pre-defined VOI modulated by experimental inputs. Using Bayesian model selection (BMS) approach (Penny et al., 2004; Stephan et al., 2009), an optimal model or a winning model is found by calculating model 'expected posterior probability' and model 'exceedance probability' of each model. Here, model expected posterior probability represents how likely it is that a model generated the data of randomly chosen subject whereas exceedance probability is a measure of degree of belief about a model having a higher posterior probability than the other remaining models (Wasserman, 2000; Stephan et al., 2010). Here, an optimal model represents the best possible combination of intrinsic and modulatory connections among pre-defined VOIs, which best explains how the observed data are generated. Further, in order to infer individual connectivity measures, another approach known as Bayesian model averaging (BMA) (Penny et al., 2010; Stephan et al., 2010) is used, which estimates a weighted average of each parameter of each model. Here, weighting of each parameter depends upon model evidence of each model.

Since the fMRI data was collected from a clinical group (stroke patients) and there was a possibility of inter-subject variability, we employed BMS and BMA using random-effects analysis (RFX) for group-level inferences (Kasess et al., 2010).

\section{Plan}

To test our previously stated hypotheses we generated eight models using $\mathrm{DCM}_{12}{ }^{1}$, for both hemispheres of all nine participants for each analysis, each session and for each task as shown in Figure $\mathbf{1}$ where 'TASK' represents tapping with either left hand (LH) (affected/unaffected), right hand $(\mathrm{RH})$ (unaffected/affected), and both hands (BH) (affected and unaffected). Theoretically, total number of models generated was 

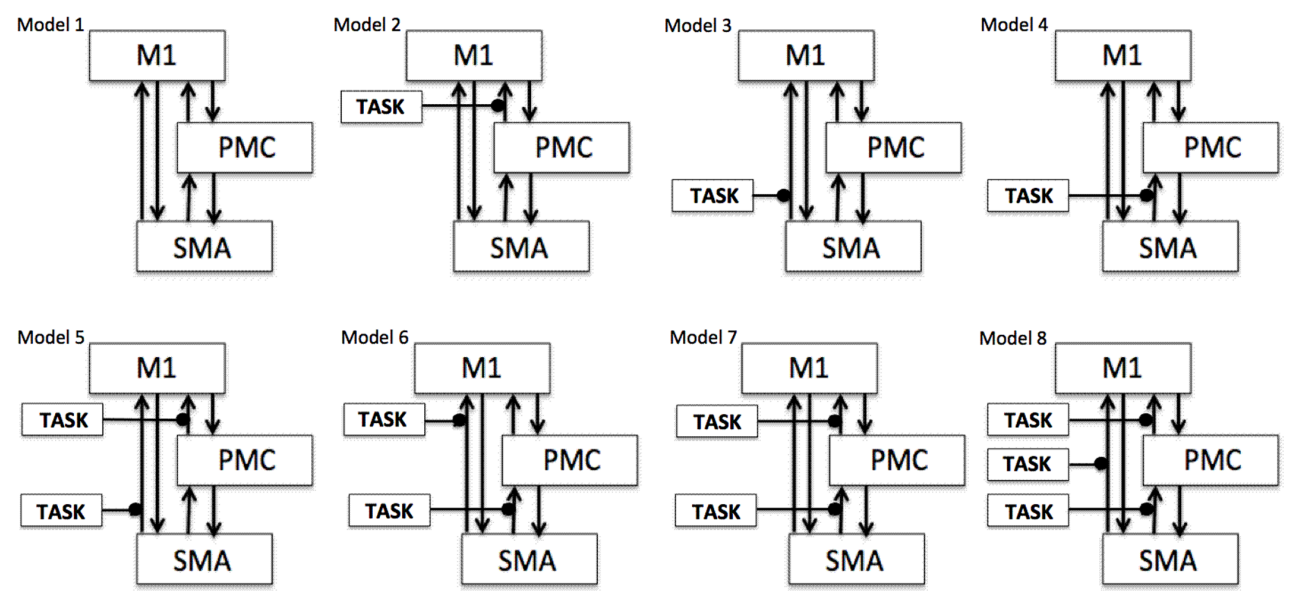

FIGURE 1 | Specification of model space. Eight models (models 1-8) are specified subject-wise, constituting bilinear family for both the hemispheres (affected and unaffected) for each analysis (AHem-aHand, AHem-bHand, UHem-aHand, and UHem-uHand) and for each session. Here 'TASK' represents 'motor execution' task, performed by either unilateral (left or right) hand or by both hands.

$576[8 \times 9$ (participants) $\times 4$ (AHem-aHand, AHem-bHand, UHem-aHand, and UHem-uHand) $\times 2$ (sessions)]. Since, one subject was excluded from the analysis and the task $\mathrm{BH}$ was included only for affected hemisphere, our model space included a total of 512 models [ $(8 \times 8$ (participants) $\times 3$ (AHem-aHand, UHem-aHand, and UHem-uHand $) \times 2$ (sessions $)+(8 \times 8$ (participants) $\times 1$ (AHem-bHand $) \times 2$ (sessions) $)]$.

We categorized our connectivity analysis into following analysis:

\section{Analysis 1: Connectivity on Affected Hemisphere Analysis 1a: AHem-aHand}

This analysis represents the condition when participants tapped with affected hand (right or left) and connectivity strength was calculated between motor areas of the affected hemisphere (left or right) (Supplementary Figures S1A,B).

Supplementary Figure S1A shows tapping with right affected hand and areas under study are on left hemisphere, which is affected. Supplementary Figure S1B shows tapping with left affected hand and right hemisphere brain areas reflect the affected hemisphere.

\section{Analysis 1b: AHem-bHand}

This analysis represents the condition when stroke survivors tapped with both hands (affected and unaffected) and connectivity strength was calculated between motor areas of the affected hemisphere (Supplementary Figures S1C,D).

Supplementary Figure S1C shows tapping with both hands (where right hand is affected) and cortical areas under study were focused on the affected- left hemisphere. Supplementary Figure S1D shows tapping with both hands (where left hand is affected) and areas under study are on right hemisphere.

\section{Analysis 2: Connectivity on Unaffected Hemisphere Analysis 2a: UHem-aHand}

This analysis represents the condition when patients tapped with affected hand (right or left) and connectivity strength was calculated between motor areas of the unaffected hemisphere (right or left) (Supplementary Figures S1E,F).

Supplementary Figure S1E shows tapping with right hand, which is affected, and areas under study are on right hemisphere, which is unaffected. Supplementary Figure S1F shows tapping with left hand, which is affected, and areas under study are on left hemisphere, which is unaffected.

\section{Analysis 2b: UHem-uHand}

This analysis represents the condition when patients tapped with their unaffected hand (left or right) and connectivity strength was calculated between motor areas of the unaffected hemisphere (right or left) (Supplementary Figures S1G,H).

Supplementary Figure S1G shows tapping with left hand, which is unaffected, and areas under study are on right hemisphere, which is unaffected. Supplementary Figure S1H shows tapping with right hand, which is unaffected, and areas under study are on left hemisphere, which is unaffected.

In Supplementary Figure S1, affected hemisphere is colored in 'red' whereas unaffected hemisphere is colored in 'gray'.

\section{RESULTS}

\section{Effective Connectivity: Optimal Model Selection}

\section{Analysis 1: Connectivity on Affected Hemisphere}

\section{Analysis 1a: AHem-aHand}

We calculated model expected $(A, C)$ and model exceedance (B,D) probability for session 1 (Figures 2A,B) and for session 2 (Figures 2C,D) for networks on affected hemisphere when patients tapped with affected hand. This showed that for AHem-aHand, model 8 was dominant with model exceedance probability of (i) 0.987 for session 1 and (ii) 0.787 for session 2 . For session 2, model 6 was the second best model with model exceedance probability of 0.175 . 
A

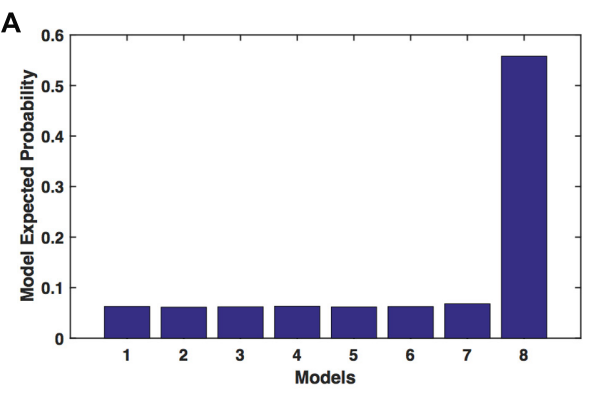

C

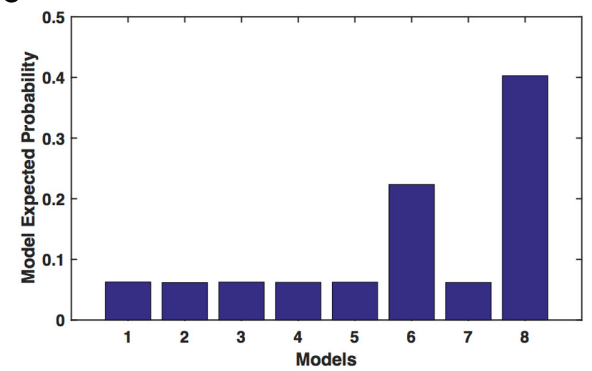

B

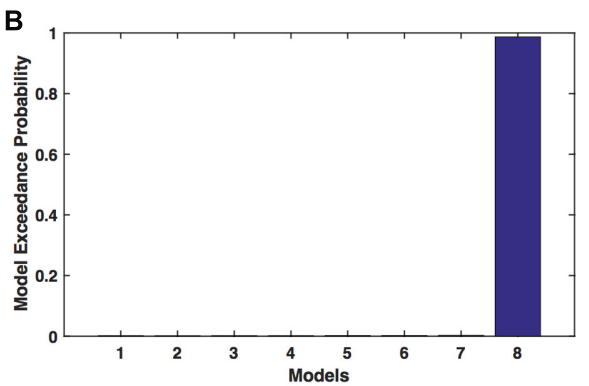

D

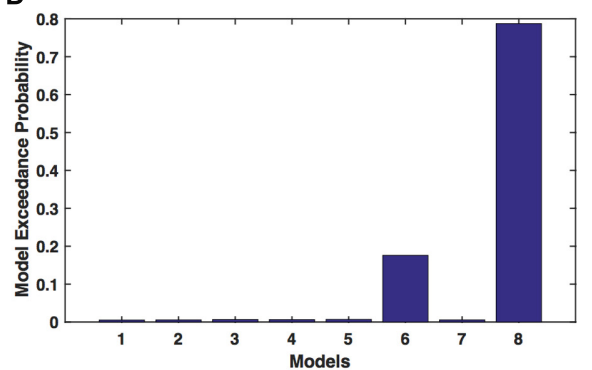

FIGURE 2 | AHem-aHand: Optimal model selection for affected hemisphere. For AHem-aHand, when participants performed the task with affected hand, model expected and model exceedance probabilities are shown for session 1 (A,B) and session 2 (C,D).

\section{Analysis 1b: AHem-bHand}

We calculated model expected $(\mathrm{A}, \mathrm{C})$ and exceedance $(\mathrm{B}, \mathrm{D})$ probability for session 1 (Figures $\mathbf{3 A}, \mathbf{B})$ and for session 2 (Figures 3C,D) for networks on affected hemisphere when patients tapped with both (affected and unaffected) hands. The analysis showed that for AHem-bHand, model 8 was dominant with model exceedance probability of (i) 0.984 for session 1 and (ii) 0.786 for session 2. For session 2, model 6 was the second best model with model exceedance probability of 0.174 .

Here, comparing BMS results for AHem-aHand and AHem$b H a n d$, we found that model 8 was dominant during during both the sessions for both the analyses. During session 2, both the analyses also showed an identical second best model as model 6.

\section{Analysis 2: Connectivity on Unaffected Hemisphere Analysis 2a: UHem-aHand}

We calculated model expected $(\mathrm{A}, \mathrm{C})$ and exceedance $(\mathrm{B}, \mathrm{D})$ probability for session 1 (Figures $4 \mathbf{A}, \mathbf{B}$ ) and for session 2 (Figures 4C,D) for networks on unaffected hemisphere when patients tapped with affected hand. This showed that for UHemaHand, model 8 was the dominant with a model exceedance probability of (i) 0.821 for session 1 and (ii) 0.534 for session 2. For session 2, model 6 had model exceedance probability of 0.359 .

Here, comparing BMS results for AHem-aHand and UHemaHand, we found that although model 8 was dominant during both sessions 1 and 2, the exceedance probability for model 8 decreased from AHem-aHand to UHem-aHand (from 0.987 to 0.821 for session 1 and from 0.787 to 0.534 for session 2) and increased for model 6 from AHem-aHand to UHem-aHand (from 0.175 to 0.359 for session 2 ).

\section{Analysis 2b: UHem-uHand}

We calculated model expected $(A, C)$ and exceedance $(B, D)$ probability for session 1 (Figures $\mathbf{5 A}, \mathbf{B}$ ) and for session 2 (Figures 5C,D) for networks on unaffected hemisphere when patients tapped with unaffected hand. This showed that for UHem-uHand, model 8 was dominant with a model exceedance probability of (i) 0.723 for session 1 and (ii) 0.542 for session 2 . For session 2, model 6 had model exceedance probability of 0.349 .

Comparing BMS results for AHem-aHand and UHem-uHand, we found that model 8 was dominant during both sessions 1 and 2 but the exceedance probability for model 8 decreased from AHem-aHand to UHem-uHand (from 0.987 to 0.723 for session 1 and from 0.787 to 0.542 for session 2) and increased for model 6 from AHem-aHand to UHem-uHand (from 0.175 to 0.349 for session 2).

When BMS results were compared for UHem-aHand and UHem-uHand, we found similar dominant network patterns during both sessions 1 and 2 . It was model 8 which was dominant with exceedance probability of (i) 0.821 for session 1 (ii) 0.534 for session 2 for UHem-aHand and (iii) 0.723 for session 1 and 0.542 for session 2 for UHem-uHand. Model 6 was the second best model for session 2 with exceedance probability of (i) 0.359 for UHem-aHand and (ii) 0.349 for UHem-uHand.

A decreasing exceedance probability occurred for model 8 during session 1 from 0.987 for AHem-aHand and 0.787 for AHem-bHand to 0.821 for UHem-aHand and further decreased to 0.723 for UHem-uHand. Similarly, an increase in exceedance probability for model 6 was found during session 2 from 0.175 for 

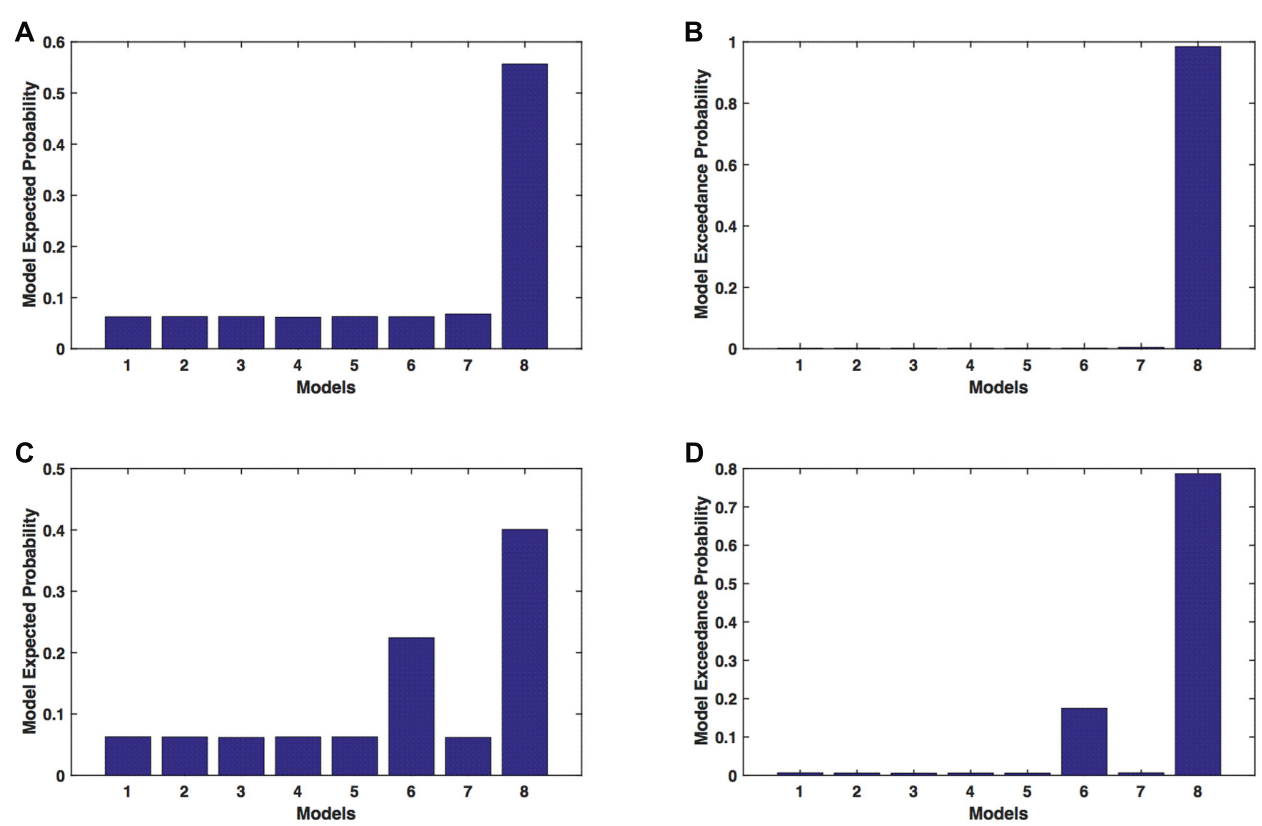

FIGURE 3 | AHem-bHand: Optimal model selection for affected hemisphere. For AHem-bHand, when participants performed the task with both (affected and unaffected) hands, model expected and model exceedance probabilities are shown for session 1 (A,B) and session 2 (C,D).

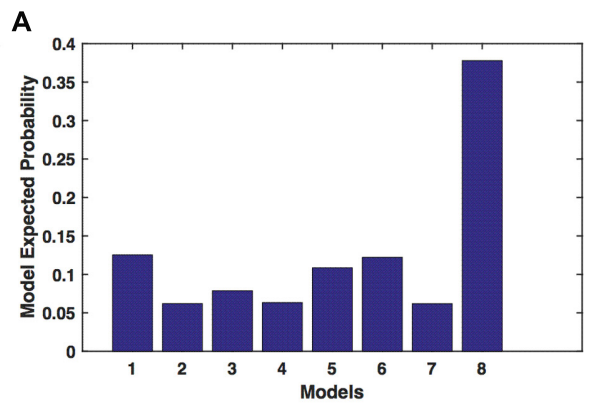

C

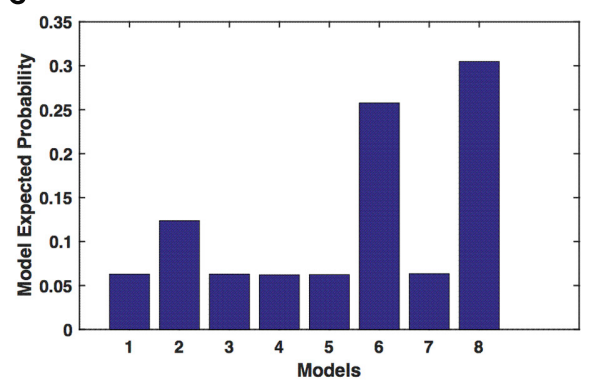

B

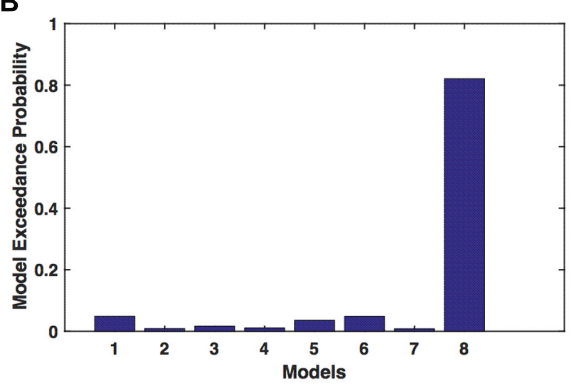

D

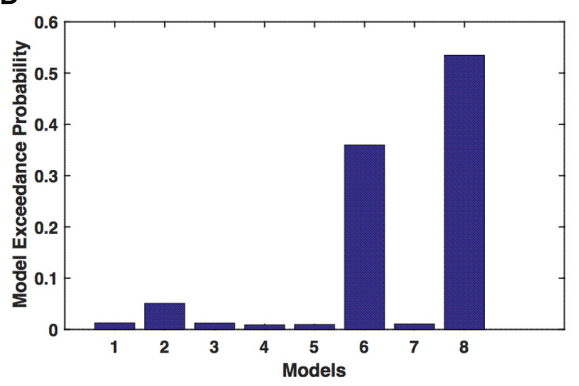

FIGURE 4 | UHem-aHand: Optimal model selection for unaffected hemisphere. For UHem-aHand, when participants performed the task with affected hand, model expected and model exceedance probabilities are shown for session 1 (A,B) and session 2 (C,D).

AHem-aHand and 0.174 for AHem-bHand to 0.359 for UHemaHand and 0.349 for UHem-uHand.

The model exceedance probabilities of the optimal models for each analysis and session calculated using BMS approach are summarized in Supplementary Table S2.

\section{Effective Connectivity: Bayesian Model Averaging (BMA)}

We used paired two-tailed t-tests to determine the significant endogenous and modulatory connections in both affected and unaffected hemisphere of motor network. 

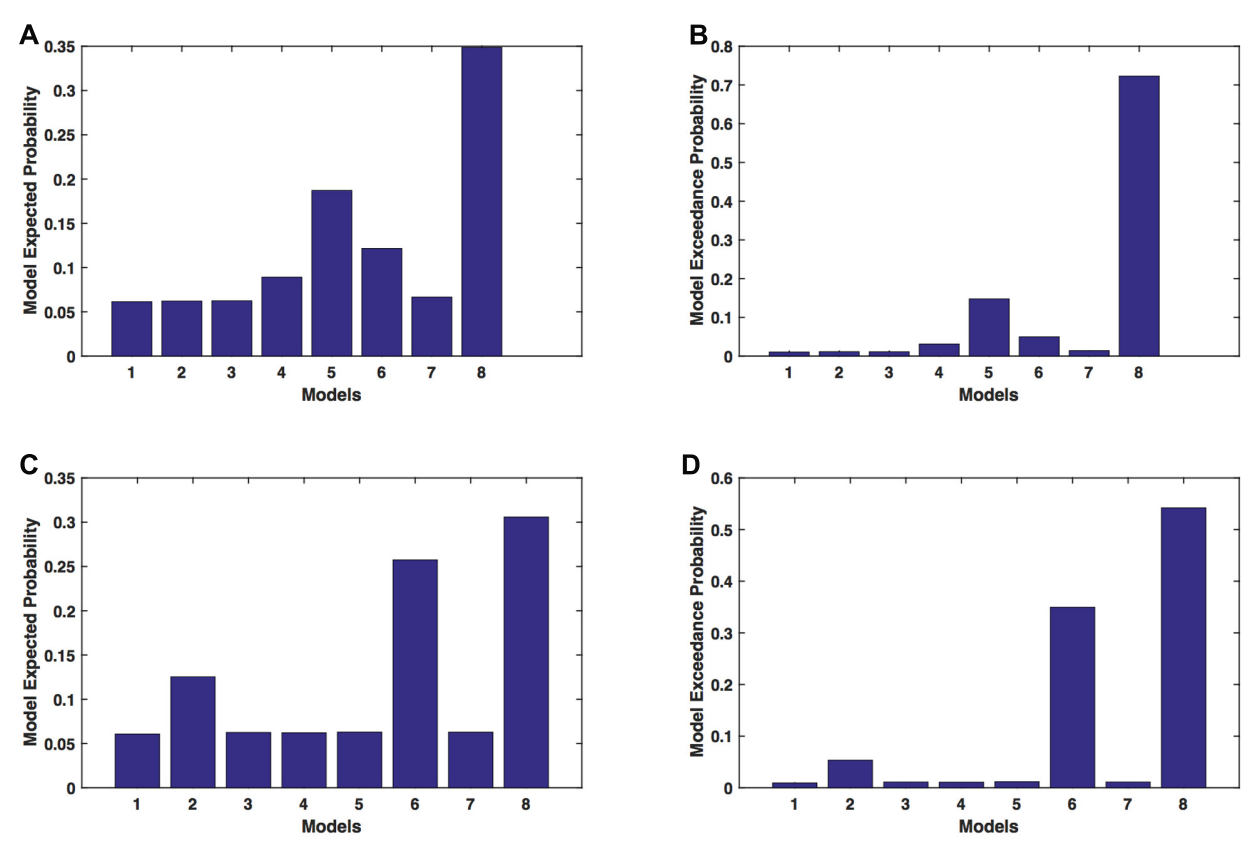

FIGURE 5 | UHem-uHand: Optimal model selection for unaffected hemisphere. For UHem-uHand, when participants performed the task with unaffected hand, model expected and model exceedance probabilities are shown for session 1 (A,B) and session 2 (C,D).

\section{AHem-aHand and AHem-bHand}

We reported significant $\left({ }^{*} p<0.001\right)$ endogenous connections (Figure 6) on the affected hemisphere when patients tapped with the affected hand (Figure 6A), i.e., AHem-aHand; both affected and unaffected hand (Figure 6B), i.e., AHem-bHand during session 1; affected hand (Figure 6C), i.e., AHem-aHand and both affected and unaffected hand (Figure 6D), i.e., AHem-bHand during session 2 .

\section{UHem-aHand and UHem-uHand}

We reported significant $\left({ }^{*} \mathrm{p}<0.001\right)$ connections (Figure 7$)$ on unaffected hemisphere when patients tapped with affected hand (Figure 7A), i.e., UHem-aHand; unaffected hand (Figure 7B), i.e., UHem-uHand during session 1; affected hand (Figure 7C), i.e., UHem-aHand and unaffected hand (Figure 7D), i.e., UHem$u$ Hand during session 2 .

When we compared individual connections for AHem-aHand, UHem-aHand, and UHem-uHand during session 2, two-tailed $t$-test showed that the connection between SMA and M1 for UHem-aHand and UHem-uHand was significantly different from AHem-aHand ( $p=0.0443$ and $p=0.0369$ for SMA to M1 and M1 to SMA, respectively, for AHem-aHand versus UHem-aHand and $p=0.0453$ and $p=0.0371$ for SMA to M1 and M1 to SMA, respectively, for AHem-aHand versus UHem-uHand but there was no significant difference between UHem-aHand and UHem-uHand for any connection.

Hence, using the BMA approach, we found that the individual connectivity between motor areas did not change on the affected hemisphere whether patients tapped with only the affected hand or with both affected as well as their unaffected hand during both the sessions 1 and 2. We also found that the bidirectional connectivity between SMA and M1 was common between UHem-aHand and UHem-uHand for both the sessions 1 and 2. Also, the bidirectional connection between SMA and M1 was significantly stronger for UHem-aHand and UHem-uHand than AHem-aHand.

The data suggest that the connectivity pattern was identical between AHem-aHand and AHem-bHand and also there was more similarity of connectivity patterns between UHem-aHand and UHem-uHand than between AHem-aHand and UHemaHand or between AHem-aHand and UHem-uHand during both the sessions 1 and 2 . We did not find any modulatory connection that was significantly stronger $(p<0.05)$ for any analysis but we certainly noticed a trend for a modulatory connection $\left({ }^{* *} p<0.1\right)$ from PMC to M1 for UHem-aHand and UHem-uHand, but not on affected hemisphere. Further, we also noticed that the individual significant connectivity differed when calculated a week apart. But $t$-tests showed that none of the connections differed significantly when compared for session 1 with session 2 for all the analyses.

Connectivity strength measures, standard deviation and significance level $\left({ }^{*} p<0.001\right.$ for endogenous connections, $* * p<0.1$ for modulatory connections) extracted using BMA for all the connections and for each analysis and each session are summarized in Supplementary Table S3.

\section{Effective Connectivity versus Clinical Scores}

We calculated the correlation between clinical scores (FMA and RMSE) and connectivity strengths (in $\mathrm{Hz}$ ) of all the connections 

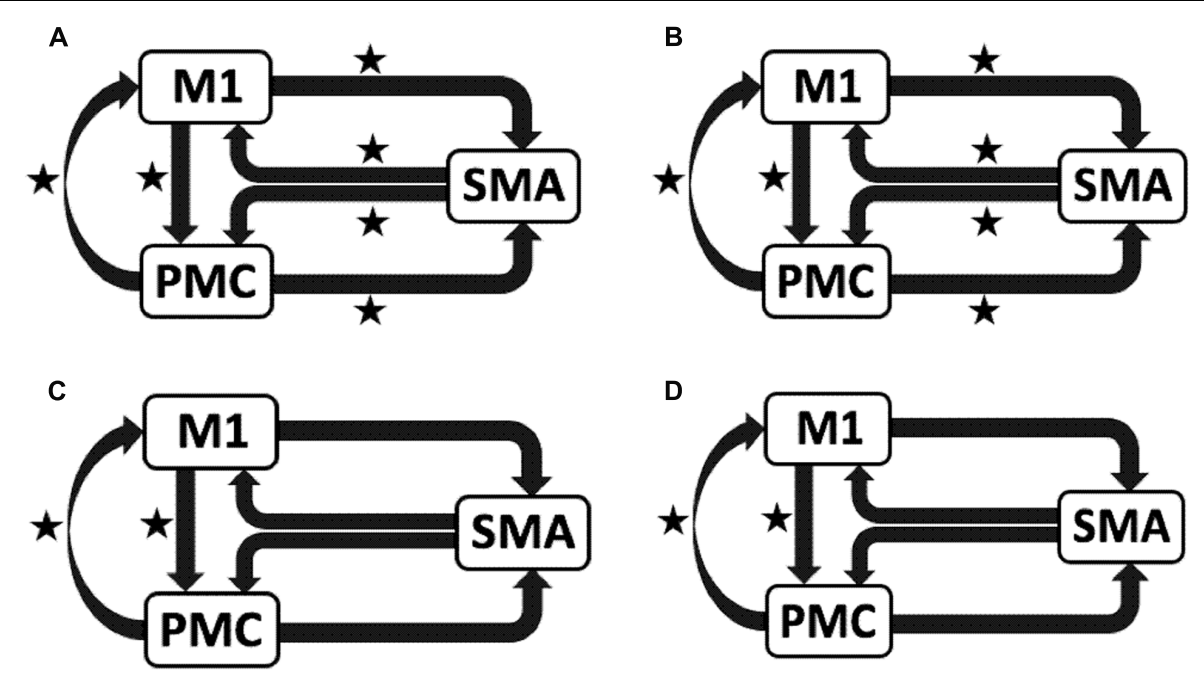

FIGURE 6 | Endogenous connectivity for affected hemisphere. Endogenous connections for AHem-aHand (when the task was performed with only affected hand) and AHem-bHand (when the task was performed with both the hands: affected and unaffected) are shown for session 1 (A,B) and session 2 (C,D). Here* represents the connections which are significantly stronger $\left({ }^{*} p<0.001, t\right.$-test).
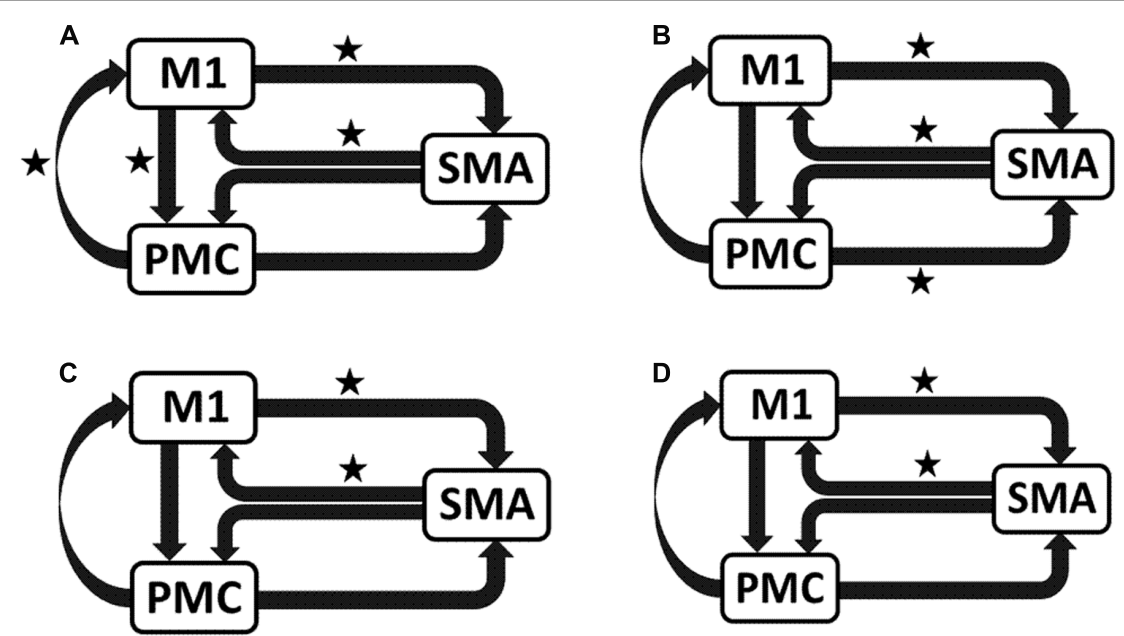

FIGURE 7 | Endogenous connectivity for unaffected hemisphere. Endogenous connections for UHem-aHand (when the task was performed with affected hand) and UHem-uHand (when the task was performed with unaffected hand) are shown for session 1 (A,B) and session 2 (C,D). Here * represents the connections which are significantly stronger $\left({ }^{*} p<0.001, t\right.$-test).

for each analysis and session using Pearson's linear correlation coefficient test. We found that for session 1, the correlation between connectivity strength between SMA and PMC and FMA score was not significant $(p>0.05)$ for AHem-aHand (Figure 8A). But there was a significant correlation $(p<0.05)$ for UHem-aHand (Figure 8B). The correlation between connectivity strength from SMA to PMC and from SMA to M1 and RMSE score was not significant $(r=-0.256, p=0.676)$ for AHem-aHand, session 1 and was positively significant $(r=0.884$, $p=0.046$ ) for AHem-aHand, session 2, respectively, (Figure 8C). Alternatively, the correlation between connectivity strength from SMA to PMC and from SMA to M1 and RMSE score was negative $(r=-0.934, p=0.020)$ for UHem-aHand, session 1 and was not significant ( $r=0.096, p=0.877$ ) for UHem-aHand, session 2, respectively (Figure 8D). Here higher FMA scores and lower RMSE scores represent better patient performance and vice-versa.

Hence, by correlating individual connectivity strengths and clinical scores for AHem-aHand and UHem-aHand for both the sessions 1 and 2, we observed significant positive correlation between brain and behavior measures (FMA scores) for two connections for UHem-aHand during session 1. We also found that RMSE scores had no negative correlation with brain connectivity measures for AHem-aHand but there was negative correlation between RMSE scores and connectivity measures for UHem-aHand. 


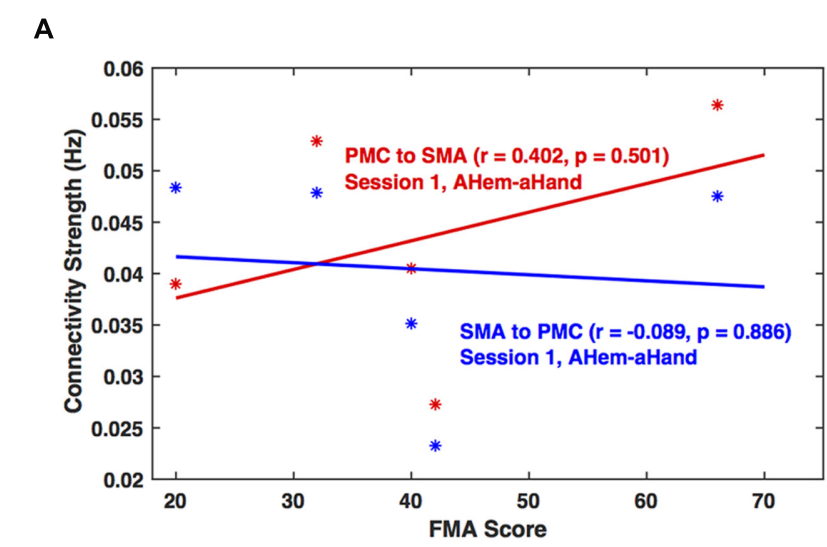

C

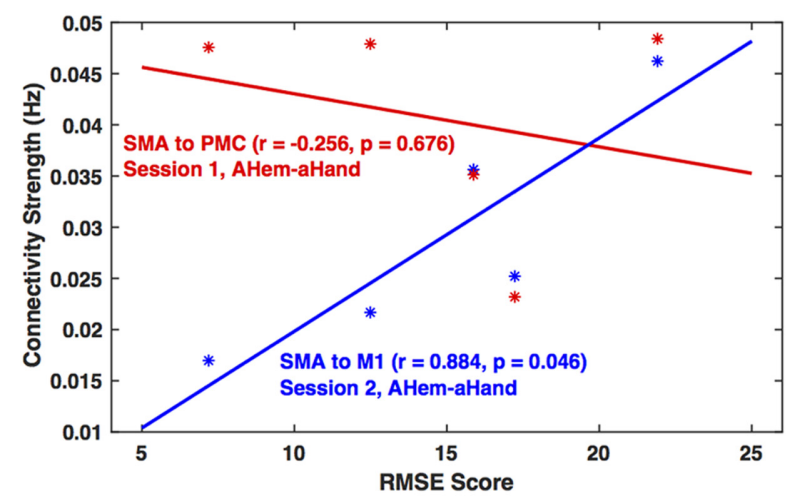

B

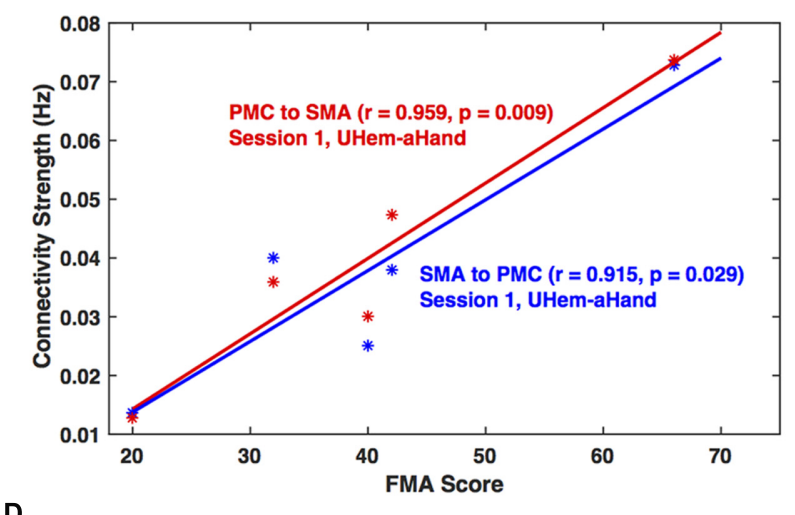

D

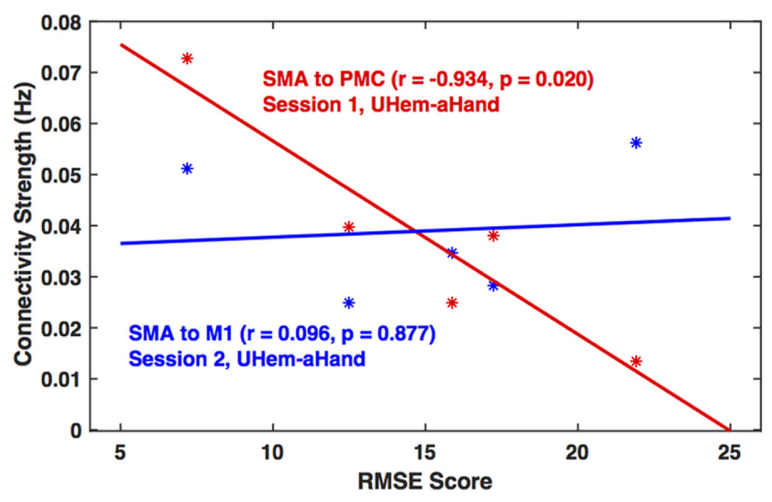

FIGURE 8 | Brain versus behavioral correlation. Brain connectivity strength measures (in $\mathrm{Hz}$ ) are plotted with the Fugl-Meyer Motor Assessment (FMA) scores $(\mathbf{A}, \mathbf{B})$ and root means square error (RMSE) (C,D) for AHem-aHand $\mathbf{( A , C )}$ and UHem-aHand (B,D).

\section{DISCUSSION}

In this study, we explored and compared the role of the affected and unaffected hemisphere during hand motor execution tasks in chronic stroke survivors. We also performed the test-retest reliability of connectivity measures between motor-areas of both hemispheres. Comparing BMS results for AHem-aHand and AHem-bHand, we found that (a) the network pattern did not change on the affected hemisphere whether patients tapped with only the affected hand or with both their affected as well as their unaffected hand during both the sessions 1 and 2 and (b) the network pattern was unchanged when analyzed a week apart for both the AHem-aHand and AHem-bHand. Further, we found that when a person who has had a stroke tapped with their affected hand, the network connectivity pattern on their unaffected hemisphere dominated over the affected hemisphere. The network pattern resembled the form of their unaffected hemisphere when they tapped with their unaffected hand. This suggests that the so-called unaffected hemisphere of stroke survivors was influenced when they tapped with their affected hand. Additionally, these observed connectivity strength characteristics appear to be stable across two observations one week apart. These findings not only reflect the reliability of fMRI technique but also of the effective connectivity approach (DCM12). From brain versus behavior (FMA and RMSE) co-relation, we observed that the connectivity pattern on unaffected hemisphere more accurately reflected the connectivity measures than the connectivity pattern on affected hemisphere when stroke patients tapped with affected hand.

\section{Contribution of Unaffected and Affected Hemispheres}

In this study, we found that the unaffected hemisphere better reflected the connectivity measures than affected hemisphere when a motor task was performed with affected hand, whereas the network pattern remained the same on the affected hemisphere independent of whether the task was performed with affected hand or with both hands. When correlating brain connectivity with measures of clinical motor behavior we found that during one of the sessions, the connectivity strength between SMA and PMC on the unaffected hemisphere was significantly (positively) correlated with FMA scores when patients performed the task with their affected hand. Also during one of the sessions, the connectivity strength from SMA to PMC on the unaffected hemisphere was found to be negatively correlated $(p=0.020)$ with RMSE scores when patients performed the task with affected hand. These correlations between brain 
networks and behavior measures reflect the fact that the connectivity pattern on unaffected hemisphere more accurately reflect the connectivity measures when patients tapped with their affected hand than the connectivity pattern of the affected hemisphere when patients tapped with their affected hand. These findings also confirmed our findings from both BMS and BMA approaches that it is the unaffected hemisphere of stroke survivors that better reflects the connectivity pattern than the affected hemisphere during a motor execution task.

In a study of eight well-recovered stroke patients, Riecker et al. (2010) studied the contribution of affected and unaffected hemisphere during motor-recovery. The authors found a linear correlation between hemodynamic responses from the affected as well as the unaffected premotor motor cortex and sensorimotor cortex and frequency of finger movements. Although this study supported the idea of bi-hemispheric recruitment in stroke survivors, their data was confounded by the use of wellrecovered stroke survivors. Additionally, the authors did not include a direct comparison of the involvement of affected and unaffected hemispheres. Using transcranial direct current stimulation, Fregni et al. (2005) explored the significance of the unaffected hemisphere for a better motor recovery. In this study, it was suggested that increased activity in the affected hemisphere can enhance the motor recovery but excessive activity in the unaffected hemisphere may provide an inadequate environment for motor recovery. So a properly balanced bihemispheric modulation of brain tissues was recommended in order to effectively promote the motor recovery. Reorganization of motor-output was also explored for the unaffected hemisphere of stroke patients (Netz et al., 1997). In this study, the motoroutputs from the unaffected hemisphere were significantly changed after stroke, although the brain activation was not correlated with clinical improvement. This study supported the idea of plastic changes in the unaffected hemisphere during motor output organization. In a recent diffusion tensor imaging (DTI) study (Jang et al., 2016), fractional anisotropy (FA) and apparent diffusion coefficient (ADC) values of the corticospinal tract (CST) were reported to be unchanged for the unaffected hemisphere but fiber volume of CST in the unaffected hemisphere was found to be increased (Kwak et al., 2010; Jang et al., 2016). There are very few studies, which report a decrease in network activity on the unaffected hemisphere. Dong and colleagues found an increase in cortical activation of $\mathrm{M} 1$ in the unaffected hemisphere but a correlation was also observed between decreased cortical activation and restoration of motor function (Dong et al., 2006). On the other hand, less affected brain side was also reported to create an abnormal activation pattern which limits the normal activation environment in the motor-network (Takeuchi and Izumi, 2012). In order to limit this maladaptive neural plasticity, specific rehabilitation techniques such as noninvasive brain stimulation were also proposed (Fregni et al., 2005; Takeuchi and Izumi, 2012).

Furthermore, comparing the contribution of both hemispheres, our study demonstrated the dominance of unaffected hemisphere over affected hemisphere when the task was performed with the affected hand. We also found that the network pattern of the unaffected hemisphere when the task was performed with the affected hand resembled the network pattern of the unaffected hemisphere when the task was performed with the unaffected hand. Hence, these findings suggested that the dominance of unaffected hemisphere might reflect a compensatory hub of connections after stroke. These results also indicate a complex functional behavior of motor networks in stroke patients. Results from previous studies are in accordance with our findings whereas a few studies reported controversial outcomes. While behavioral improvement were found to be in close association with sensorimotor networks consisting of medial PMC, lateral PMC, primary motor cortex and primary somatosensory cortices in the affected hemisphere (Pineiro et al., 2002; Loubinoux et al., 2003; Calautti et al., 2007; Loubinoux, 2007), functional reorganization within unaffected hemisphere was found to be supporting motor recovery (O'Shea et al., 2007; Riecker et al., 2010; Rehme et al., 2011). Besides these reports, in a study of five patients, increased cortical activation was reported in the unaffected hemisphere during movement of the affected hand at less than 25 days after onset of the stroke but the activation was found to be normal at 35 days after onset (Takeda et al., 2007). A more detailed description of the changes in functional network after stroke was provided by $\mathrm{Li}$ et al. (2014) where they reported that some of the damaged connections of functional network could be compensated by new indirect connections or circuits produced after stroke (Li et al., 2014). Hence, these studies confirmed our findings that the unaffected hemisphere always had a role to play after stroke by dominating and compensating the role of affected hemisphere.

\section{Test-Retest Reliability}

After comparing the exceedance probabilities of Bayesian models after a week apart, we found that the dominant network was consistent for (i) the affected hemisphere when the task was performed with either the affected hand or with both the hands and (ii) the unaffected hemisphere when the task was performed with either the affected hand or unaffected hand. Furthermore, by comparing the individual connectivity strengths calculated using the BMA approach, we did not find any connections, which differ significantly measured one week apart.

From several years, fMRI has emerged as one of the most popular and commonly used technique to study brain activations and for advanced-level connectivity analysis. Studies like fMRI reliability with time play a significant role in identifying an effective and reliable stroke rehabilitation technique among several. Previously, several studies reported the reliability of BOLD fMRI signals over time in healthy people during a variety of tasks such as cognitive, sensorimotor, working memory, motor-imagery, and execution tasks (Loubinoux et al., 2001; Aron et al., 2006; Lim et al., 2007; Yoo et al., 2007; Kimberley et al., 2008a,b; Caceres et al., 2009). Several other studies on healthy controls even reported very high variability in the magnitude and spatial extent of activations when comparison 
was done between sessions (Miki et al., 2000; Loubinoux et al., 2001; Liu et al., 2004). These studies suggested that the reliability of fMRI is yet to be confirmed even for healthy controls. It can be imagined how controversial fMRI results could be for reliability studies of impaired individuals and for people suffering from more complex brain disorders. Further, network and individual connectivity analysis might further add variability to the connectivity results and can originate more controversial findings based on fMRI technique.

Further to translate neuronal activity to BOLD signal, neuronal model implemented in DCM is considered as one of the most appropriate models as it is more flexible with the shape of hemodynamic response function (HRF) (Schuyler et al., 2010). Schuyler et al. (2010) also reported that brain activation and its interactions were more reliable when connectivity information was considered in comparison to when only effects of general linear model (GLM) were considered. DCM was also considered to be very sensitive to the nuances of fMRI signal changes and a fair to excellent scan-rescan DCM reliability was reported (Schuyler et al., 2010). However, the test-retest reliability of DCM for fMRI was again in question recently when different versions of DCM were tested. Classical DCM in SPM5 was found be more reliable than DCM10 in SPM8 for fMRI (Frässle et al., 2015). But in another study of face perception network by Frässle et al. (2016), stable conventional activity and effective connectivity measures were obtained using BMS and parameter estimation approach.

\section{CONCLUSION}

Results of the present study uncovered (i) an important brainbehavior relationship between the connectivity in the unaffected brain hemisphere and the motor behavior of the affected hands in stroke patients and (ii) the test-retest reliability of fMRI and effective connectivity approach. Findings reported in this study strengthened our understanding of stroke conditions and brain plasticity in stroke survivors. We believe that such studies play a crucial role identifying an effective stroke rehabilitation therapy. Future studies with a larger sample size and involvement of agematched healthy controls would further enhance the importance of unaffected hemisphere and its role while treating stroke patients.

\section{REFERENCES}

Aron, A. R., Gluck, M. A., and Poldrack, R. A. (2006). Long-term test-retest reliability of functional MRI in a classification learning task. Neuroimage 29, 1000-1006. doi: 10.1016/j.neuroimage.2005.08.010

Arya, K. N., Pandian, S., Verma, R., and Garg, R. K. (2011). Movement therapy induced neural reorganization and motor recovery in stroke: a review. J. Bodyw. Mov. Ther. 15, 528-537. doi: 10.1016/j.jbmt.2011.01.023

Ashburner, J., and Friston, K. J. (1999). Nonlinear spatial normalization using basis functions. Hum. Brain Mapp. 7, 254-266. doi: 10.1002/(SICI)1097-0193(1999) 7:4<254::AID-HBM4>3.3.CO;2-7

Bajaj, S., Butler, A. J., Drake, D., and Dhamala, M. (2015a). Brain effective connectivity during motor-imagery and execution following stroke and rehabilitation. Neuroimage 8, 572-582. doi: 10.1016/j.nicl.2015.06.006

\section{AUTHOR CONTRIBUTIONS}

Conceived and designed the experiments: DW, GJ, AB. Performed the experiments: SB, DW, AB. Analyzed the data: SB, SH. Wrote the paper: SB, SH, MD, GJ, AB.

\section{ACKNOWLEDGMENTS}

This study was supported by 5I21RX000561 (B7676-P) U.S. Department of Veterans Affairs, Rehabilitation Research \& Development Service to AB. The authors would also like to thank Dr. Daniel Drake and Kimberly Richards for their assistance in data collection.

\section{SUPPLEMENTARY MATERIAL}

The Supplementary Material for this article can be found online at: http://journal.frontiersin.org/article/10.3389/fnhum. 2016.00650/full\#supplementary-material

\section{TABLE S1 | Demographics, stroke locations and behavioral scores for stroke survivors.}

TABLE S2 | Optimal model selection. Model excedance probabilities of optimal models for each case and session calculated using Bayesian model selection (BMS) approach are summarized.

TABLE S3 | Bayesian model averaging (BMA) parameters. Connectivity strength measures, standard deviation and significance level $\left({ }^{*} p<0.001\right.$ for endogenous connections, ${ }^{* *} p<0.1$ for modulatory connections, $t$-test) extracted using Bayesian model averaging (BMA) for all the connections and for each case and each session are summarized.

FIGURE S1 | AHem-aHand, AHem-bHand, UHem-aHand, and UHem-uHand. Here we summarize all the four cases of connectivity analysis. (A-D): Here 'affected' hemisphere, colored as 'red' is under study when stroke survivors performed the task with affected hand i.e., either right affected hand (A) or left affected hand (B) (AHem-aHand) and with both the hands i.e., with either right affected and left unaffected (C) or left affected and right unaffected (D) (AHem-bHand). (E,F): Here 'unaffected' hemisphere, colored as 'gray' is under study when stroke survivors performed the task with affected hand i.e., either right affected hand (E) or left affected hand (F) (UHem-aHand). (G,H): Here 'unaffected' hemisphere, colored as 'gray' is under study when stroke survivors performed the task with unaffected hand i.e., either left unaffected hand (G) or right unaffected hand $\mathbf{( H )}$ (UHem-uHand).

Bajaj, S., Butler, A. J., Drake, D., and Dhamala, M. (2015b). Functional organization and restoration of the brain motor-execution network after stroke and rehabilitation. Front. Hum. Neurosci. 9:174. doi: 10.3389/fnhum.2015. 00173

Bajaj, S., Drake, D., Butler, A. J., and Dhamala, M. (2014). Oscillatory motor network activity during rest and movement: an fNIRS study. Front. Syst. Neurosci. 8:13. doi: 10.3389/fnsys.2014.00013

Bajaj, S., Lamichhane, B., Adhikari, B. M., and Dhamala, M. (2013). Amygdala mediated connectivity in perceptual decision-making of emotional facial expressions. Brain Connect. 3, 386-397. doi: 10.1089/brain. 2013.0145

Brewer, L., Horgan, F., Hickey, A., and Williams, D. (2013). Stroke rehabilitation: recent advances and future therapies. QJM 106, 11-25. doi: 10.1093/qjmed/ hcs 174 
Brott, T., Adams, H. P., Olinger, C. P., Marler, J. R., Barsan, W. G., Biller, J., et al. (1989). Measurements of acute cerebral infarction: a clinical examination scale. Stroke 20, 864-870. doi: 10.1161/01.STR.20.7.871

Buetefisch, C. M. (2015). Role of the contralesional hemisphere in post-stroke recovery of upper extremity motor function. Front. Neurol. 6:214. doi: 10.3389/ fneur.2015.00214

Bütefisch, C. M., Kleiser, R., Körber, B., Müller, K., Wittsack, H. J., Hömberg, V., et al. (2005). Recruitment of contralesional motor cortex in stroke patients with recovery of hand function. Neurology 64, 1067-1069. doi: 10.1212/01.WNL. 0000154603.48446 .36

Caceres, A., Hall, D. L., Zelaya, F. O., Williams, S. C., and Mehta, M. A. (2009). Measuring fMRI reliability with the intra-class correlation coefficient. Neuroimage 45, 758-768. doi: 10.1016/j.neuroimage.2008.12.035

Calautti, C., Naccarato, M., Jones, P. S., Sharma, N., Day, D. D., Carpenter, A. T., et al. (2007). The relationship between motor deficit and hemisphere activation balance after stroke: a 3T fMRI study. Neuroimage 34, 322-331. doi: 10.1016/j. neuroimage.2006.08.026

Cárdenas-Morales, L., Volz, L. J., Michely, J., Rehme, A. K., Pool, E. M., Nettekoven, C., et al. (2014). Network connectivity and individual responses to brain stimulation in the human motor system. Cereb. Cortex 24, 1697-1707. doi: 10.1093/cercor/bht023

Chen, J. L., and Schlaug, G. (2013). Resting state interhemispheric motor connectivity and white matter integrity correlate with motor impairment in chronic stroke. Front. Neurol. 4:178. doi: 10.3389/fneur.2013.00178

Colebatch, J. G., and Gandevia, S. C. (1989). The distribution of muscular weakness in upper motor neuron lesions affecting the arm. Brain 112, 749-763. doi: $10.1093 /$ brain/112.3.749

Dobkin, B. H. (2004). Strategies for stroke rehabilitation. Lancet Neurol. 3, 528-536. doi: 10.1016/S1474-4422(04)00851-8

Dong, Y., Dobkin, B. H., Cen, S. Y., Wu, A. D., and Winstein, C. J. (2006). Motor cortex activation during treatment may predict therapeutic gains in paretic hand function after stroke. Stroke 37, 1552-1555. doi: 10.1161/01.STR. $0000221281.69373 .4 \mathrm{e}$

Doyon, J., and Benali, H. (2005). Reorganization and plasticity in the adult brain during learning of motor skills. Curr. Opin. Neurobiol. 15, 161-167. doi: 10. 1016/j.conb.2005.03.004

Fong, K. N., Chan, C. C., and Au, D. K. (2001). Relationship of motor and cognitive abilities to functional performance in stroke rehabilitation. Brain Injury 15, 443-453. doi: 10.1080/02699050010005940

Frässle, S., Paulus, F. M., Krach, S., and Jansen, A. (2016). Test-retest reliability of effective connectivity in the face perception network. Hum. Brain Mapp. 37, 730-744. doi: 10.1002/hbm.23061

Frässle, S., Stephan, K. E., Friston, K. J., Steup, M., Krach, S., Paulus, F. M., et al. (2015). Test-retest reliability of dynamic causal modeling for fMRI. Neuroimage 117, 56-66. doi: 10.1016/j.neuroimage.2015.05.040

Fregni, F., Boggio, P. S., Mansur, C. G., Wagner, T., Ferreira, M. J., Lima, M. C., et al. (2005). Transcranial direct current stimulation of the unaffected hemisphere in stroke patients. Neuroreport 16, 1551-1555. doi: 10.1097/01.wnr.0000177010. $44602.5 \mathrm{e}$

Friston, K. J., Harrison, L., and Penny, W. (2003). Dynamic causal modelling. Neuroimage 19, 1273-1302. doi: 10.1016/S1053-8119(03)00202-7

Fugl-Meyer, A. R., Jaasko, L., Leyman, I., Olsson, S., and Steglind, S. (1975). The post-stroke hemiplegic patient. 1. A method for evaluation of physical performance. Scand. J. Rehabil. Med. 7, 13-31.

Gentili, R., Han, C. E., Schweighofer, N., and Papaxanthis, C. (2010). Motor learning without doing: trial-by-trial improvement in motor performance during mental training. J. Neurophysiol. 104, 774-783. doi: 10.1152/jn.00257. 2010

Gladstone, D. J., Danells, C. J., and Black, S. E. (2002). The Fugl-Meyer assessment of motor recovery after stroke: a critical review of its measurement properties. Neurorehabil. Neural Repair 16, 232-240. doi: 10.1177/154596802401105171

Grefkes, C., and Fink, G. R. (2011). Reorganization of cerebral networks after stroke: new insights from neuroimaging with connectivity approaches. Brain 134, 1264-1276. doi: 10.1093/brain/awr033

Guo, C. C., Kurth, F., Zhou, J., Mayer, E. A., Eickhoff, S. B., Kramer, J. H., et al. (2012). One-year test-retest reliability of intrinsic connectivity network fMRI in older adults. Neuroimage 61, 1471-1483. doi: 10.1016/j.neuroimage.2012. 03.027
Haaland, K. Y., and Harrington, D. L. (1989). Hemispheric control of the initial and corrective components of aiming movements. Neuropsychologia 27, 961-969. doi: 10.1016/0028-3932(89)90071-7

Haaland, K. Y., Prestopnik, J. L., Knight, R. T., and Lee, R. R. (2004). Hemispheric asymmetries for kinematic and positional aspects of reaching. Brain 127, 1145-1158. doi: 10.1093/brain/awh133

Jang, S. H., Yi, J. H., Choi, B. Y., Chang, C. H., Jung, Y. J., Lee, H. D., et al. (2016). Changes of the corticospinal tract in the unaffected hemisphere in stroke patients: a diffusion tensor imaging study. Somatosens. Mot. Res. 33, 1-7. doi: 10.3109/08990220.2016.1142435

John, C., Pablo, A. C., and Gregory, T. C. (2015). Stroke Rehabilitation. Amsterdam: Elsevier.

Jones, R. D., Donaldson, I. M., and Parkin, P. J. (1989). Impairment and recovery of ipsilateral sensory-motor function following unilateral cerebral infarction. Brain 112, 113-132. doi: 10.1093/brain/112.1.113

Kasess, C. H., Stephan, K. E., Weissenbacher, A., Pezawas, L., Moser, E., and Windischberger, C. (2010). Multi-subject analyses with dynamic causal modeling. Neuroimage 49, 3065-3074. doi: 10.1016/j.neuroimage.2009. 11.037

Kimberley, T. J., Birkholz, D. D., Hancock, R. A., Vonbank, S. M., and Werth, T. N. (2008a). Reliability of fMRI during a continuous motor task: assessment of analysis techniques. J. Neuroimag. 18, 18-27. doi: 10.1111/j.1552-6569.2007. 00163.x

Kimberley, T. J., Khandekar, G., and Borich, M. (2008b). fMRI reliability in subjects with stroke. Exp. Brain Res. 186, 183-190. doi: 10.1007/s00221-007-1221-8

Kwak, S. Y., Yeo, S. S., Choi, B. Y., Chang, C. H., and Jang, S. H. (2010). Corticospinal tract change in the unaffected hemisphere at the early stage of intracerebral hemorrhage: a diffusion tensor tractography study. Eur. Neurol. 63, 149-153. doi: 10.1159/000281108

Li, J., Liu, J., Liang, J., Zhang, H., Zhao, J., Rieth, C. A., et al. (2010). Effective connectivities of cortical regions for top-down face processing: a dynamic causal modeling study. Brain Res. 1340, 40-51. doi: 10.1016/j.brainres.2010.04. 044

Li, W., Li, Y., Zhu, W., and Chen, X. (2014). Changes in brain functional network connectivity after stroke. Neural Regen. Res. 9, 51-60. doi: 10.4103/1673-5374. 125330

Li, Y., Wang, D., Zhang, H., Wang, Y., Wu, P., Zhang, H., et al. (2016). Changes of brain connectivity in the primary motor cortex after subcortical stroke: a multimodal magnetic resonance imaging study. Medicine (Baltimore) 95: e2579. doi: 10.1097/MD.0000000000002579

Lim, J., Choo, W. C., and Chee, M. W. (2007). Reproducibility of changes in behaviour and $\mathrm{fMRI}$ activation associated with sleep deprivation in a working memory task. Sleep 30, 61-70.

Lindenberg, R., Zhu, L. L., Rüber, T., and Schlaug, G. (2012). Predicting functional motor potential in chronic stroke patients using diffusion tensor imaging. Hum. Brain Mapp. 33, 1040-1051. doi: 10.1002/hbm.21266

Liu, J. Z., Zhang, L., Brown, R. W., and Yue, G. H. (2004). Reproducibility of fMRI at $1.5 \mathrm{~T}$ in a strictly controlled motor task. Magn. Reson. Med. 52, 751-760. doi: $10.1002 / \mathrm{mrm} .20211$

Lorist, M. M., Kernell, D., Meijman, T. F., and Zijdewind, I. (2002). Motor fatigue and cognitive task performance in humans. J. Neurophysiol. 545, 313-319.

Lotze, M., Markert, J., Sauseng, P., Hoppe, J., Plewnia, C., and Gerloff, C. (2006). The role of multiple contralesional motor areas for complex hand movements after internal capsular lesion. J. Neuroscience 26, 6096-6102. doi: 10.1523/ JNEUROSCI.4564-05.2006

Loubinoux, I. (2007). Can fMRI measures of brain motor activation add significantly to other variables in the prediction of treatment response? Stroke 38, 2032-2033. doi: 10.1161/STROKEAHA.107.488908

Loubinoux, I., Carel, C., Alary, F., Boulanouar, K., Viallard, G., Manelfe, C., et al. (2001). Within-session and between-session reproducibility of cerebral sensorimotor activation: a test-retest effect evidenced with functional magnetic resonance imaging. J. Cereb. Blood Flow Metab. 21, 592-607. doi: 10.1097/ 00004647-200105000-00014

Loubinoux, I., Carel, C., Pariente, J., Dechaumont, S., Albucher, J. F., Marque, P., et al. (2003). Correlation between cerebral reorganization and motor recovery after subcortical infarcts. Neuroimage 20, 2166-2180. doi: 10.1016/j. neuroimage.2003.08.017 
Mazziotta, J. C., Toga, A. W., Evans, A., Fox, P., and Lancaster, J. (1995). A Probablistic atlas of the human brain: theory and rationale for its development. Neuroimage 2, 89-101. doi: 10.1006/nimg.1995.1012

Miki, A., Raz, J., Van Erp, T. G., Liu, C. S., Haselgrove, J. C., and Liu, G. T. (2000). Reproducibility of visual activation in functional MR imaging and effects of postprocessing. Am. J. Neuroradiol. 21, 910-915.

Netz, J., Lammers, T., and Hömberg, V. (1997). Reorganization of motor output in the non-affected hemisphere after stroke. Brain 120, 1579-1586. doi: 10.1093/ brain/120.9.1579

O'Shea, J., Johansen-Berg, H., Trief, D., Göbel, S., and Rushworth, M. F. (2007). Functionally specific reorganization in human premotor cortex. Neuron 54, 479-490. doi: 10.1016/j.neuron.2007.04.021

Park, C. H., Chang, W. H., Ohn, S. H., Kim, S. T., Bang, O. Y., PascualLeone, A., et al. (2011). Longitudinal changes of resting-state functional connectivity during motor recovery after stroke. Stroke 42, 1357-1362. doi: 10.1161/STROKEAHA.110.596155

Parker Jones, O., Seghier, M. L., Kawabata Duncan, K. J., Leff, A. P., Green, D. W., and Price, C. J. (2013). Auditory-motor interactions for the production of native and non-native speech. J. Neurosci. 33, 2376-2387. doi: 10.1523/ JNEUROSCI.3289-12.2013

Penny, W. D., Stephan, K. E., Daunizeau, J., Rosa, M. J., Friston, K. J., Thomas, M., et al. (2010). Comparing families of dynamic causal models. PLoS Comput. Biol. 6:e1000709. doi: 10.1371/journal.pcbi.1000709

Penny, W. D., Stephan, K. E., Mechelli, A., and Friston, K. J. (2004). Comparing dynamic causal models. Neuroimage 22, 1157-1172. doi: 10.1016/j.neuroimage. 2004.03.026

Pineiro, R., Pendlebury, S., Johansen-Berg, H., and Matthews, P. M. (2002). Altered hemodynamic responses in patients after subcortical stroke measured by functional MRI. Stroke 33, 103-109. doi: 10.1161/hs0102.100482

Rehme, A. K., Fink, G. R., Von Cramon, D. Y., and Grefkes, C. (2011). The role of the contralesional motor cortex for motor recovery in the early days after stroke assessed with longitudinal FMRI. Cereb. Cortex 21, 756-768. doi: 10.1093/cercor/bhq140

Riecker, A., Gröschel, K., Ackermann, H., Schnaudigel, S., Kassubek, J., and Kastrup, A. (2010). The role of the unaffected hemisphere in motor recovery after stroke. Hum. Brain Mapp. 31, 1017-1029. doi: 10.1002/hbm.20914

Sanford, J., Moreland, J., Swanson, L. R., Stratford, P. W., and Gowland, C. (1993). Reliability of the Fugl-Meyer assessment for testing motor performance in patients following stroke. Phys. Ther. 73, 447-454.

Schaechter, J. D., and Perdue, K. L. (2008). Enhanced cortical activation in the contralesional hemisphere of chronic stroke patients in response to motor skill challenge. Cereb. Cortex 18, 638-647. doi: 10.1093/cercor/bhm096

Schuyler, B., Ollinger, J. M., Oakes, T. R., Johnstone, T., and Davidson, R. J. (2010). Dynamic Causal Modeling applied to fMRI data shows high reliability. Neuroimage 49, 603-611. doi: 10.1016/j.neuroimage.2009.07.015

Schwarz, A. J., and McGonigle, J. (2011). Negative edges and soft thresholding in complex network analysis of resting state functional connectivity data. Neuroimage 55, 1132-1146. doi: 10.1016/j.neuroimage.2010.12.047
Seitz, R. J., Höflich, P., Binkofski, F., Tellmann, L., Herzog, H., and Freund, H. J. (1998). Role of the premotor cortex in recovery from middle cerebral artery infarction. Arch. Neurol. 55, 1081-1088. doi: 10.1001/archneur.55. 8.1081

Stephan, K. E., Penny, W. D., Daunizeau, J., Moran, R. J., and Friston, K. J. (2009). Bayesian model selection for group studies. Neuroimage 46, 1004-1017. doi: 10.1016/j.neuroimage.2009.03.025

Stephan, K. E., Penny, W. D., Moran, R. J., Den Ouden, H. E., Daunizeau, J., and Friston, K. J. (2010). Ten simple rules for dynamic causal modeling. Neuroimage 49, 3099-3109. doi: 10.1016/j.neuroimage.2009.11.015

Takeda, K., Gomi, Y., Imai, I., Shimoda, N., Hiwatari, M., and Kato, H. (2007). Shift of motor activation areas during recovery from hemiparesis after cerebral infarction: a longitudinal study with near-infrared spectroscopy. Neurosci. Res. 59, 136-144. doi: 10.1016/j.neures.2007.06.1466

Takeuchi, N., and Izumi, S. (2012). Maladaptive plasticity for motor recovery after stroke: mechanisms and approaches. Neural Plast. 2012:359728.

Telesford, Q. K., Morgan, A. R., Hayasaka, S., Simpson, S. L., Barret, W., Kraft, R. A., et al. (2010). Reproducibility of graph metrics in FMRI networks. Front. Neuroinform. 4:117. doi: 10.3389/fninf.2010.00117

Ward, N. S., Brown, M. M., Thompson, A. J., and Frackowiak, R.S. J. (2003). Neural correlates of motor recovery after stroke: a longitudinal fMRI study. Brain 126, 2476-2496. doi: 10.1093/brain/awg245

Wasserman, L. (2000). Bayesian model selection and model averaging. J. Math. Psychol. 44, 92-107. doi: 10.1006/jmps.1999.1278

Weiller, C., Chollet, F., Friston, K. J., Wise, R. J., and Frackowiak, R. S. (1992). Functional reorganization of the brain in recovery from striatocapsular idarction in man. Ann. Neurol. 31, 463-472. doi: 10.1002/ana.4103 10502

Winstein, C. J., and Pohl, P. S. (1995). Effects of unilateral brain damage on the control of goal-directed hand movements. Exp. Brain Res. 105, 163-174. doi: $10.1007 / \mathrm{BF} 00242191$

Wulf, G., and Schmidt, R. A. (1997). Variability of practice and implicit motor learning. J. Exp. Psychol. 23, 987-1006.

Yoo, S. S., O’leary, H. M., Lee, J. H., Chen, N. K., Panych, L. P., and Jolesz, F. A. (2007). Reproducibility of trial-based functional MRI on motor imagery. Int. J. Neurosci. 117, 215-227. doi: 10.1080/00207450600582546

Conflict of Interest Statement: The authors declare that the research was conducted in the absence of any commercial or financial relationships that could be construed as a potential conflict of interest.

Copyright (c) 2016 Bajaj, Housley, Wu, Dhamala, James and Butler. This is an open-access article distributed under the terms of the Creative Commons Attribution License (CC BY). The use, distribution or reproduction in other forums is permitted, provided the original author(s) or licensor are credited and that the original publication in this journal is cited, in accordance with accepted academic practice. No use, distribution or reproduction is permitted which does not comply with these terms. 